\title{
Porosity Determination of Carbon and Glass Fibre Reinforced Polymers Using Phase-Contrast Imaging
}

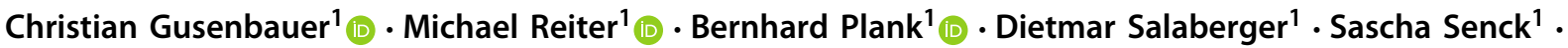 \\ Johann Kastner ${ }^{1}$ iD
}

Received: 5 April 2018 / Accepted: 28 September 2018 / Published online: 3 November 2018

(c) The Author(s) 2018

\begin{abstract}
This paper presents multi-modal image data of different fibre reinforced polymer samples acquired with a desktop Talbot-Lau grating interferometer (TLGI) X-ray computed tomography (XCT) system and compare the results with images acquired using conventional absorption-based XCT. Two different fibre reinforced polymer samples are investigated: (i) a carbon fibre reinforced polymer (CFRP) featuring a copper mesh embedded near the surface for lightning conduction and (ii) a short glass fibre reinforced polymer (GFRP) sample. The primary goal is the non-destructive detection of internal defects such as pores and the quantification of porosity. TLGI provides three imaging modalities including attenuation contrast (AC) due to absorption, differential phase contrast (DPC) due to refraction and dark-field contrast (DFC) due to scattering. In the case of the CFRP sample, DPC is less prone to metal streak artefacts improving the detection of pores that are located close to metal components. In addition, results of a metal artefact reduction (MAR) method, based on sinogram inpainting and an image fusion concept for AC, DPC and DPC, are presented. In the case of the GFRP sample, DPC between glass fibres and matrix is lower compared to AC while DPC shows an increased contrast between pores and its matrix. Porosity for the CFRP sample is determined by applying an appropriate global thresholding technique while an additional background removal is necessary for the GFRP sample.
\end{abstract}

Keywords X-ray computed tomography · Talbot-Lau grating interferometer · Differential phase contrast · Carbon and glass fibre reinforced polymers · Porosity

\section{Introduction}

X-ray computed tomography (XCT) based on absorption contrast (AC) is an essential imaging technique in order to

Christian Gusenbauer

christian.gusenbauer@fh-wels.at

Michael Reiter

michael.reiter@fh-wels.at

Bernhard Plank

bernhard.plank@fh-wels.at

Dietmar Salaberger

dietmar.salaberger@fh-wels.at

Sascha Senck

sascha.senck@fh-wels.at

Johann Kastner

johann.kastner@fh-ooe.at

1 University of Applied Sciences Upper Austria, Stelzhamerstraße 23, 4600 Wels, Austria reveal internal structures. However, certain material combinations lead to strong artefacts that degrade image quality and may conceal defects in critical areas. Conventional XCT may also struggle in the case of low absorbing materials or materials with similar attenuation coefficients. Within the last 10 years an innovative $\mathrm{X}$-ray technology based on the TalbotLau effect [1-3] has become available in laboratory research, extending the imaging capabilities of conventional laboratory XCT systems that are restricted to the single imaging modality of AC. Talbot-Lau grating interferometer (TLGI) $\mathrm{XCT}$ introduces two additional imaging modalities: differential phase contrast (DPC) due to refraction and dark-field contrast (DFC) due to scattering. This method is continuously evolving by improved grating design [4], advanced reconstruction techniques [5] and image processing routines [6], while suitable materials science applications have been identified [7-10]. Up to now, DFC turns out to be the key imaging modality for materials science, since it is capable of revealing interface contrast of internal structures and defects such as 
small pores, gaps or cracks in the sub-voxel region that can be even smaller than the spatial resolution of the XCT system $[11,12]$. DFC is highly sensitive to the size of internal scattering objects allowing quantitative studies for a given material and interferometer setup [13]. In addition, DFC enables the extraction of information about microstructural anisotropy, since scattering information from the internal microstructure (e.g. differently orientated fibres [9] or fibre bundles [7]) is direction-dependent. DFC's anisotropy in combination with the extraction of sub-voxel microstructure can be used to characterize anisotropic materials such as fibre reinforced polymers with a larger field of view as compared to AC imaging. This has been realised by different techniques such as X-ray vector radiography [14] or X-ray tensor tomography [15] based on combining an imaging setup with multiple sample orientations. By applying stitching modes, large fields of view up to $0.5 \mathrm{~m}^{2}$ can be investigated in radioscopic mode as well [16]. In contrast to that, DPC has proven its usefulness by improved diagnostic capabilities compared to conventional methods [17], but so far there is a rather low impact on materials science applications.

This paper shows the advantages and benefits of DPC Xray imaging applied to different fibre reinforced polymer systems. We demonstrate that DPC images show significantly decreased beam-hardening (streaking) artefacts in a multi-material system with copper mesh and an increased contrast-to-noise ratio, facilitating pore segmentation. Nevertheless, when dealing with a carbon fibre reinforced polymer material system without any metallic components, conventional absorption-based XCT is still the most appropriate characterization technique for porosity evaluations [18].

\section{Experimental Methods}

\subsection{Fibre Reinforced Polymeric Samples}

In this paper we characterize carbon and glass fibre reinforced polymer samples using a desktop TLGI system. The maximum sample size is $20 \mathrm{~mm}$ in diameter and $60 \mathrm{~mm}$ in length. The maximum tube voltage is $60 \mathrm{kV}$. Due these restriction samples were cut out from a real part (door of an airplane) to be able to mount the sample inside the chamber and to ensure specimen penetration by X-rays in the lower $\mathrm{kV}$ range. The carbon fibre reinforced polymer (CFRP) sample features a copper mesh near the surface to ease lightening conduction [19]. It consists of two layups, multiple prepreg layers made of woven carbon fibre bundles (Hexply F584), which are bonded by an adhesive film. This adhesive film has a higher absorption contrast compared to the epoxy matrix. The process of manufacture is the common curing under $3-5$ atm pressure under vacuum at $180{ }^{\circ} \mathrm{C}$. Important material characteristics of the CFRP sample are the distribu- tion and orientation of the fibre bundles and the number and distribution of pores that are caused by trapped volatiles in the resin. The diameter of the individual carbon fibres is in the range of 6-7 $\mu \mathrm{m}$ and the diameter of the copper wires is about $90 \mu \mathrm{m}$. The sample dimensions are $9 \mathrm{~mm} \times 4 \mathrm{~mm}$ (width $\times$ thickness). The scanned height is about $15 \mathrm{~mm}$ for the SkyScan 1294 device and about $9 \mathrm{~mm}$ for the Nanotom $180 \mathrm{NF}$ device. Achievable voxel sizes are in the range of 5-20 $\mu \mathrm{m}$.

The glass fibre reinforced polymer (GFRP) sample consists of a polypropylene (PP) matrix filled with 32 weight percent of short glass fibres (PPGF32). Therefore a multipurpose test specimens ISO type A was manufactured by an injection moulding process. For CT-inspection, the sample dimensions were reduced to $5 \mathrm{~mm} \times 4 \mathrm{~mm}$ (width $\mathrm{x}$ thickness) with a scanned height of about $5 \mathrm{~mm}$. Important material characteristics of the GFRP sample are the distribution and orientation of the fibres as well as the number and distribution of pores. The diameter of the individual glass fibres is in the range of $10-12 \mu \mathrm{m}$.

\subsection{Conventional Absorption-Based XCT}

$\mathrm{X}$-rays are used to generate 2D images of objects typically on digital detectors that are sensitive to radiation by placing the specimen between the X-ray source and detector (see Fig. 1a). Polychromatic X-rays penetrate the specimen and are absorbed in accordance with Lambert-Beer's law. The energy of the X-ray spectra as well as thickness, elemental composition and density of the object influence the attenuation and produce a $2 \mathrm{D}$ projection image of the object on the detector. Defects show as attenuation changes in the acquired images. For tomographic operation, a set of $2 \mathrm{D}$ projection images is acquired during a full rotation of the specimen. The complete series of projection images allows the reconstruction of the three-dimensional distribution of the spatial X-ray attenuation. The result of the reconstruction is a volumetric grey-value dataset consisting of volumetric pixels (voxels). For reference measurements a conventional absorption-based Nanotom 180 NF XCT device (GE Measurement \& Control) was used (see Fig. 1a). Scanning parameters are presented in Table 1.

\subsection{Talbot-Lau Grating Interferometer XCT}

An illustration of the working principle of a typical TLGI XCT setup is depicted in Fig. 1b, showing three additional gratings G0, G1 and G2 that are placed in the optical beam path between source and detector in comparison to a conventional XCT system (see Fig. 1a). A movable source grating (G0) creates an array of line sources, ensuring sufficient transverse coherence length, while a diffractive grating (G1) causes phase modulation of the incident X-rays resulting 


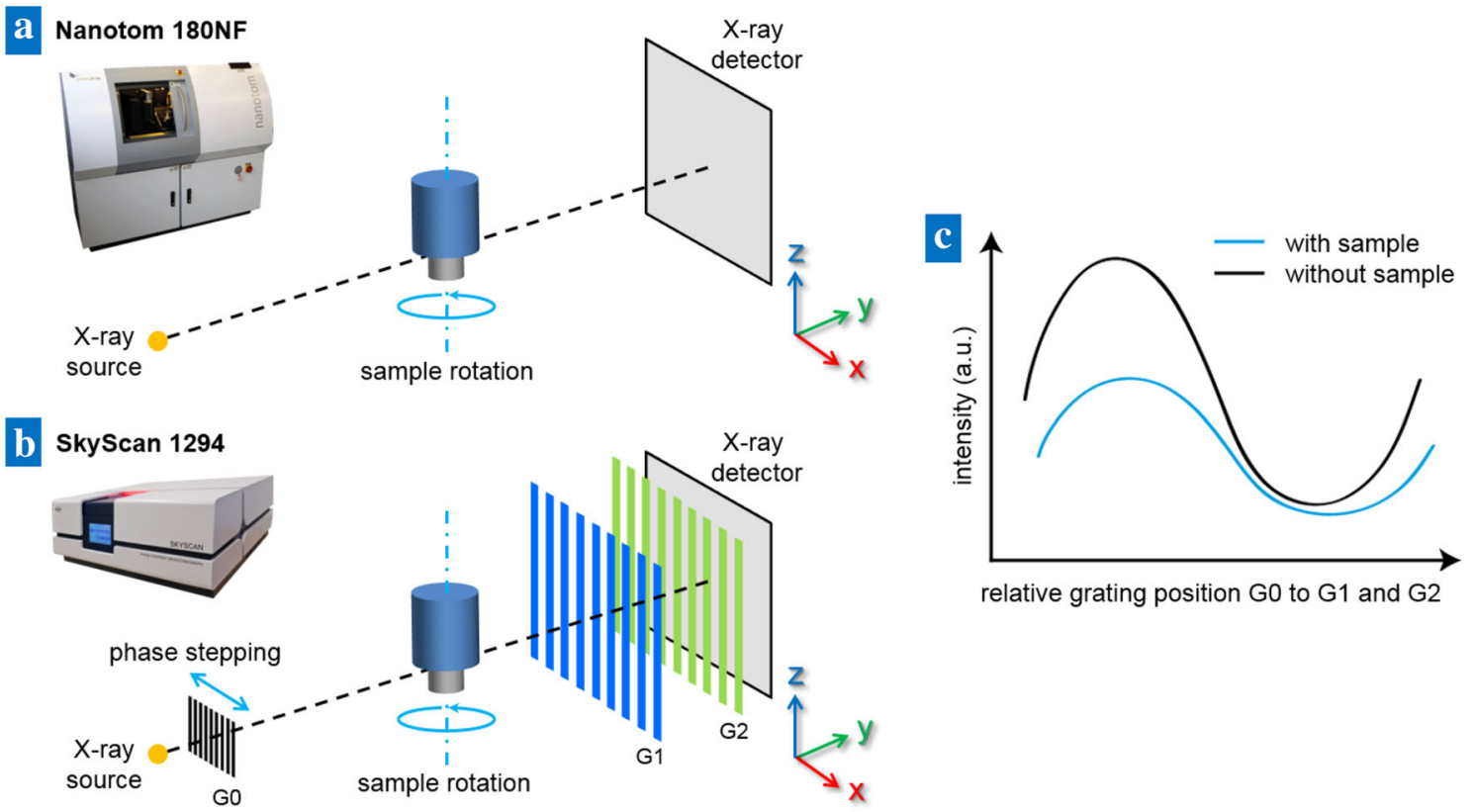

Fig. 1 Illustration of the working principle of a a conventional XCT system and b a TLGI setup with source grating G0, phase grating G1 and absorption grating G2; $\mathbf{c}$ phase stepping approach as a schematic graph of the sinusoidal intensity modulations for one detector pixel with and without object

Table 1 XCT scanning parameters

\begin{tabular}{|c|c|c|c|c|c|}
\hline XCT systems & Image modalities & Sample & $\begin{array}{l}\text { Scanning parameters } \\
\text { (tube voltage and } \\
\text { pre-filter) }\end{array}$ & Voxel size & $\begin{array}{l}\text { Exposure } \\
\text { time }\end{array}$ \\
\hline \multirow[t]{2}{*}{ Talbot-Lau $\mu$ XCT (SkyScan 1294) } & $\mathrm{AC}, \mathrm{DPC}, \mathrm{DFC}$ & $\mathrm{CFRP} / \mathrm{Cu}$ & $35 \mathrm{kV}, \mathrm{Al} 0.25 \mathrm{~mm}$ & $(22.8 \mu \mathrm{m})^{3}$ & $780 \mathrm{~min}$ \\
\hline & & GFRP & $35 \mathrm{kV}, \mathrm{Al} 0.25 \mathrm{~mm}$ & $(22.8 \mu \mathrm{m})^{3}$ & $195 \min$ \\
\hline High-resolution $\mu \mathrm{XCT}$ (Nanotom $180 \mathrm{NF}$ ) & $\begin{array}{c}\text { Conventional AC } \\
\text { reference scan }\end{array}$ & $\mathrm{CFRP} / \mathrm{Cu}$ & $95 \mathrm{kV}, \mathrm{Al} 0.2 \mathrm{~mm}$ & $(5 \mu \mathrm{m})^{3}$ & $106 \min$ \\
\hline
\end{tabular}

in an interference pattern. Since the fringe pattern cannot be resolved directly with conventional X-ray detectors, an absorption grating (G2) is placed in front of the detector [3]. G1 and G2 represent the interferometer part of the TLGI system. The Talbot-Lau effect $[20,21]$ causes the self-imaging of a grating via diffraction in the near field and via interfering waves at regular Talbot distances. A phase stepping procedure of the source grating G0 is used to scan transversely across the repeated intensity pattern at the position of G2, resulting in a sinusoidal intensity modulation for each detector pixel at the detector plane (see Fig. 1c) with and without an object. Rotating the sample stepwise in between the acquisition of the phase stepping curves allows the tomographic operation.

A sample placed within the optical beam axis between source, gratings and detector will attenuate, refract, and scatter the incident X-ray depending on the material phases, sample properties and microstructure and thus perturbing the interference pattern. $\mathrm{AC}$ is formed due to the attenuation of photons that partially pass through the object. DPC is related to the index of refraction and its image contrast is the result of local deflections of the X-ray beam. DFC is formed by changes in the total amount of radiation that is scattered at small angles by microscopic inhomogeneities in the sample in forward direction (e.g. by particles, pores, and fibers) [22]. A Fourier analysis of the intensity modulations of each detector pixel is used to simultaneously extract co-registered AC, DPC, and DFC images.

For this study a desktop TLGI XCT system (SkyScan 1294, see Fig. 1b) from Bruker microCT using a phase stepping approach was used to characterize and evaluate the CFRP and GFRP samples by extracting AC, DPC and DFC imaging modalities and to determine the respective porosity. The Talbot-Lau data acquired from the desktop system has been partly reconstructed by the SkyScan 1294 software (NRecon), as well as by a phase-weighted filtered back projection (FBP) algorithm using the image reconstruction software by the company Mitos. Scanning parameters 
including tube voltage, voxel size and exposure time are presented in Table 1. Exposure time is calculated by multiplying the number of projections, the integration time, the number of phase steps and frame averaging. Previous studies have shown that maximum visibility for the given sample geometries can be achieved with $35 \mathrm{kV}$ and a pre-filtration of $0.25 \mathrm{~mm}$ of $\mathrm{Al}$, since this particular parameter combination fits best with the design energy of the desktop TLGI system [23, 24]. The design energy is a setup-dependent value characterizing the energy at which maximum visibility is achieved. Visibility is a common measure for the additional imaging modalities DPC and DFC. It can be expressed as the ratio of Fourier components a1 and a0 extracted from the periodic intensity modulations [25], since the change in oscillation amplitude (visibility) is an important performance parameter of the interferometer as it is a direct measure for the degree of the interference. A decrease in visibility contrast for higher energies has been reported for the desktop TLGI system [23], since increasing transparency of the grating bars (with increasing energy) lowers grating efficiency, resulting in an increasing mismatch between design energy of the system and the effective energy of the applied X-ray spectra. Since the average energy behind the object is higher due to beam hardening effects, an additional mismatch might lower the maximum visibility further.

\subsection{Metal Artefact Reduction (MAR)}

In multi-material components, non-linearities in the projection data caused by, for example, beam hardening and scattered radiation can lead to severe artefacts in the reconstructed XCT images. These artefacts are seen as dark or bright streaks that may conceal material inhomogeneities such as pores, gaps or cracks in critical areas of the specimen. There are two possible strategies to reduce these artefacts and to improve image quality: (i) using additional pre-filter material during scanning or (ii) applying suitable correction methods that are based for example on sinogram manipulation. The use of pre-filtration typically leads to longer scanning times to compensate for the loss of photons. In this work, a metal artefact reduction (MAR) method based on sinogram inpainting was applied to the reference AC scan data of the CFRP sample with copper mesh, since it is capable of reducing streaking artefacts originating from metallic components. MAR-corrected results are compared to DPC data in order to discuss the benefits of DPC imaging applied to multi-material components.

The original MAR method [26] relies on the detection and deletion of the so-called metal trace within the sinogram by segmenting metal parts in an initial reconstructed volume. The segmented metal parts are then forward-projected in order to identify metal areas in the original sinogram. These areas are removed from the sinogram and the missing data is restored by for example linear interpolation. Meyer et al. proposed the normalized MAR (NMAR) [27] correction method based on an improved interpolation, since the interpolation in the original MAR method may cause blurring of edges or the formation of streaks due to interpolation inaccuracies, e.g. if the transition between original and interpolated projection data is not smooth enough. The NMAR approach introduces an additional normalization step of the projections before and after interpolation by dividing and multiplying a sinogram of a prior image of the matrix material to the original sinogram. This leads to a flat sinogram, which can be interpolated in a smoother way. The corrected sinogram is then reconstructed and the segmented metal parts are re-inserted into the reconstructed volume data. The NMAR method has been successfully applied to medical [27] as well as industrial XCT data [28].

\section{Results and Discussion}

\subsection{Carbon Fibre Reinforced Polymer (CFRP) with Copper Mesh}

Figure 2 shows multi-modal axial slice images acquired with a conventional Nanotom 180 NF XCT system (AC data) and with the SkyScan 1294 desktop TLGI device (DPC and DFC data). Each imaging modality offers specific insights into the material system, since AC, DPC and DFC are sensitive to different material phases, properties and inhomogeneities due to different interaction mechanisms of X-rays with matter (attenuation, refraction, scattering).

Figure 2 shows the different components of the sample, the copper meshes at the surface, carbon fibre bundles, resin and pores. The vertical structure in Fig. $2 a, b$ is resin with higher density. Figure 2a shows AC data with pronounced metallic artefacts that arise due to beam hardening effects and scattered radiation. These artefacts hinder proper sample inspection and failure analysis of areas close to the metallic components. Pores in areas without artefacts can be detected with sufficient contrast. Figure $2 b$ shows the NMAR-corrected AC data with strongly reduced streaking artefacts originating from the copper mesh. Even though the overall image quality has improved, additional interpolation artefacts are introduced in the corrected data (see indications of white arrows in Fig. 2b). These interpolation artefacts strongly depend on the quality of the segmented prior image, on the amount and shape of metal parts and on the interpolation method itself. Nevertheless, the detection capabilities of pores can be greatly improved e.g. for pore positions \#1 and \#2. Pore position \#3 is also affected by sinogram manipulation and shows a slight loss in contrast. In addition, it appears that the copper mesh is embedded in a higher absorbing resin 


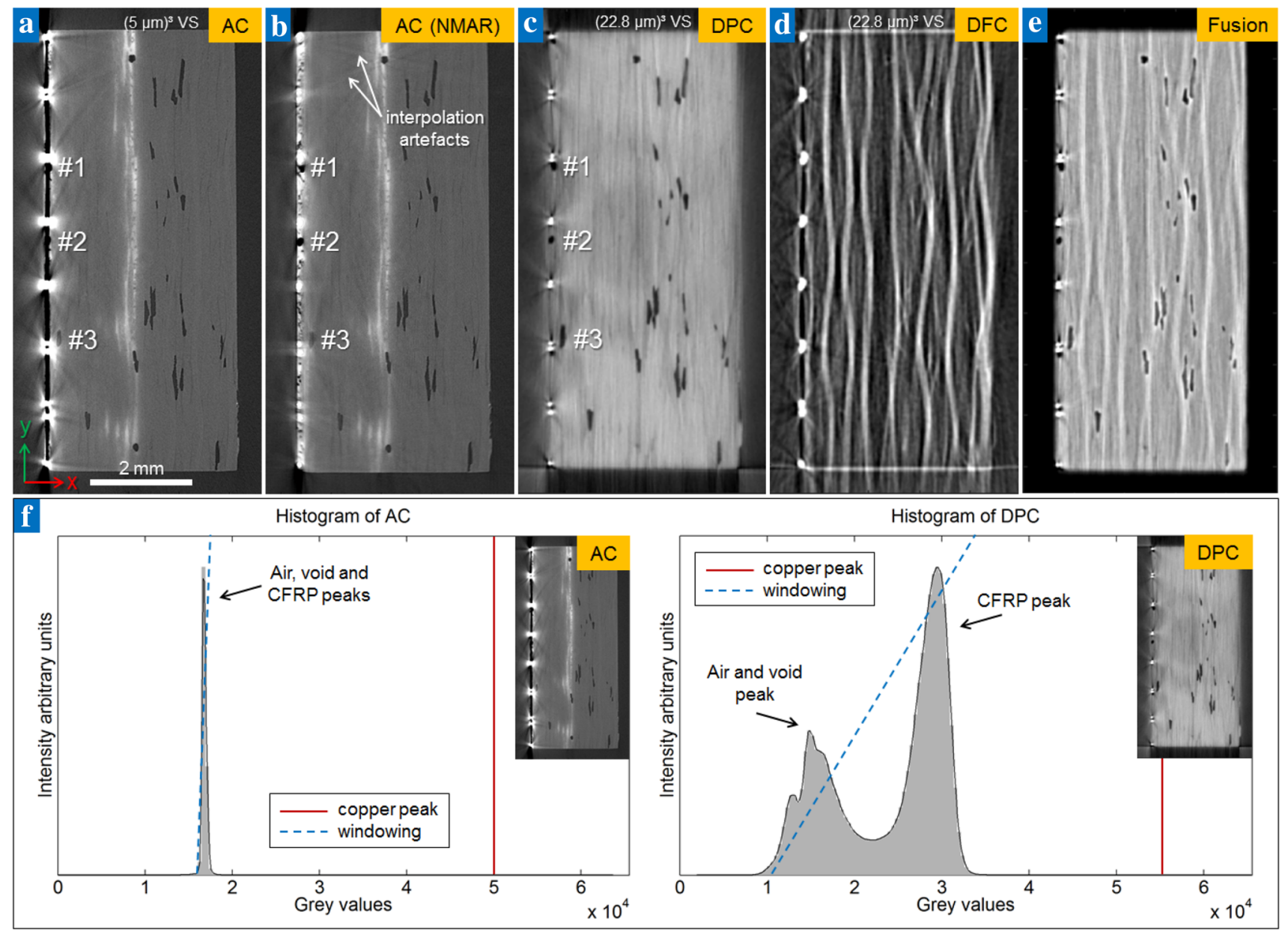

Fig. 2 Axial slice images of CFRP sample with copper mesh: a Nanotom AC data, b normalized metal artefact corrected (NMAR) Nanotom AC data, c DPC data from SkyScan 1294 and d DFC data from SkyScan $1294, \mathbf{e}$ a fusion approach of AC, DPC and DFC for displaying pores and

or adhesive layer which is not clearly visible in the uncorrected AC data.

In comparison to the uncorrected AC image, the DPC image in Fig. 2c shows almost no metallic streaking artefacts and provides an increased contrast between pores and matrix components (carbon fibres and neat epoxy resin), facilitating the pore segmentation process. This is also obvious from the histograms plotted in Fig. 2f, showing that DPC produces a slightly better discrimination between air (void) and CFRP peak (see Fig. $2 \mathrm{f}$ right) as compared to the uncorrected AC image (see Fig. $2 \mathrm{f} \mathrm{left)}$ ). Pores that are located close to or in between the copper mesh are also better visible in the corresponding DPC image as compared to the NMAR-corrected case. Figure 3, confirms this findings by zoom-in images and grey values profiles at pore \#3 (Fig. 2). Unfortunately, further quantitative analysis of the image quality by e.g. contrastto-noise ratio values is not possible, since the specimen is too in-homogenous for the definition of homogenous images fibre bundles within one single image. $\mathbf{f}$ histograms of uncorrected $\mathrm{AC}$ and DPC data showing positions of air/voids and CFRP, high absorbing copper structures are mapped to the maximum of the shown 16 bit histograms

regions that allow the calculation of meaningful contrast and noise values.

Even though the X-ray beam is heavily attenuated at positions of the copper mesh, it is still refracted and the interferometer setup is able to detect sample-induced changes of the beam directions, since the $\mathrm{X}$-ray phase shift cross section is almost a thousand times larger than the $\mathrm{X}$-ray absorption cross section [29]. In addition, the X-ray phase shift cross section shows a rather linear dependence of the X-ray energy and atomic number and thus is less prone to beam hardening effects especially for multi-material systems with large differences in attenuation coefficients. The DFC image in Fig. $2 \mathrm{~d}$ reveals individual carbon fibre bundles that are orientated perpendicular to the rotation axis [7], which cannot be resolved clearly by AC. Due to the horizontal or vertical arrangement of the grating lines, only a certain direction of fibre bundles contributes to the scatter signal. In order to reveal carbon fibre bundles that are oriented differently 

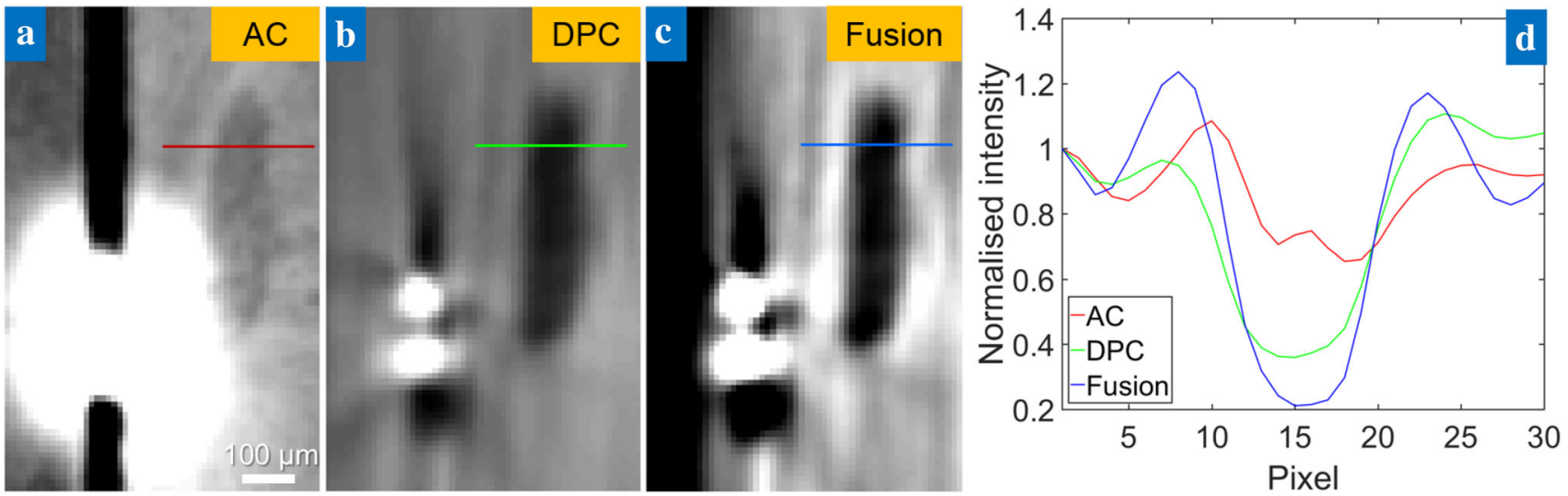

Fig. 3 Zoom in on axial slice images of the CFRP sample with copper mesh at pore \#3 (Fig. 2): a Nanotom AC data, b SkyScan DPC data and $\mathbf{c}$ the fusion result. $\mathbf{d}$ shows grey value profiles at pore \#3 extracted at the colored lines in the slice images
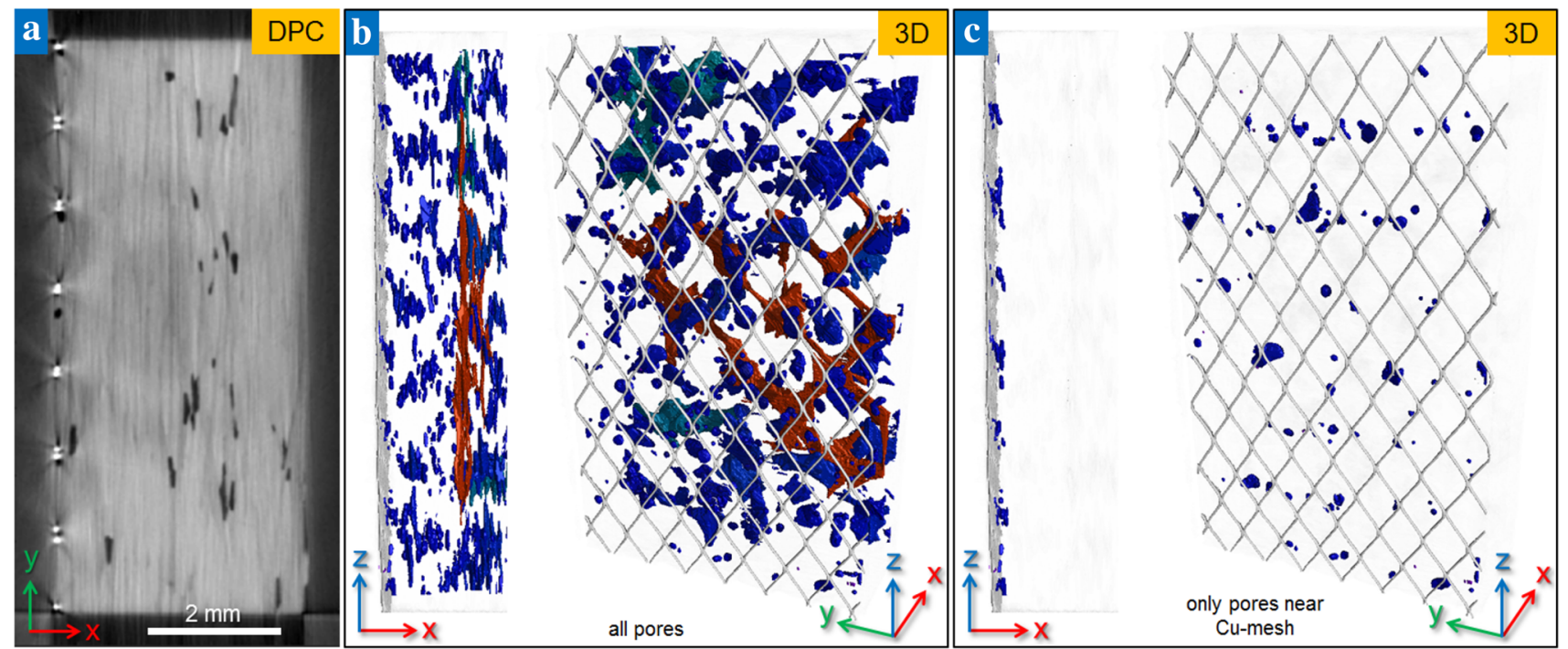

Fig. 4 CFRP sample with copper mesh: a axial slice image of DPC from SkyScan 1294 and 3D renderings along different viewing directions of DPC data with semi-transparent matrix showing pores and the $\mathrm{Cu}$-mesh for different regions of interest: $\mathbf{b}$ all pores and $\mathbf{c}$ pores close to the $\mathrm{Cu}$ mesh that would be missed when evaluating only AC data; the colour coding corresponds to the pore volume (Color figure online) within the sample volume, the sample would have to be scanned multiple times with different sample positions each time in order to acquire the complete scatter signal from different fibre bundle orientations. See for example [7, 15].

While AC, DPC and DFC are able to separate different material properties and features (metal components, fibre bundles, resin material, pores in the case of the CFRP sample), it may be useful to show all material-related features in one single image. In particular, the fused data may be helpful to detect pores (e.g. data from AC and DPC) and to directly compare pore positions with fibre bundles (data from DFC). Figure 2e shows a simple image fusion approach from a previous publication [24] that has been used to combine suitable information from different image modalities using a frequency-split method in order to combine high- pass and low-pass filtered images with proper image masks to gain local contrast enhancements for further (visual) material inspection. The fused image shows more homogenouslydistributed grey values, while retaining the sharpness and preventing the occurrence of streaking artefacts. The fused data may help the user to detect pores and to directly compare pore positions within the fibre bundles, which facilitates the inspection task and may be used to gain further insight into the material system.

Since the DPC imaging modality provides good separability between pores and matrix material and shows less metal streaking artefacts (Fig. 4a), DPC is suitable to perform porosity evaluations without needing an additional MAR correction. Figure $4 \mathrm{~b}, \mathrm{c}$ show the segmentation results in the form of $3 \mathrm{D}$ renderings with a semi-transparent matrix by 
Table 2 Porosity values of $\mathrm{CFRP} / \mathrm{Cu}$ and GFRP specimen segmented by a global ISO50 threshold method

\begin{tabular}{lllll}
\hline Material system & Imaging modality & Region-of-interest & $\begin{array}{l}\text { Defect volume in } \\
{\left[\mathrm{mm}^{3}\right]}\end{array}$ & Porosity in [\%] \\
\hline $\mathrm{CFRP} / \mathrm{Cu}$ & DPC & Whole sample & 11.10 & 2.43 \\
& & Area close to Cu & 0.42 & 0.09 \\
$\mathrm{CFRP} / \mathrm{Cu}^{*}$ & AC & $\begin{array}{c}\text { Reduced sample } \\
\text { height, area close } \\
\text { to Cu excluded }\end{array}$ & 7.62 & 3.09 \\
& & & 6.63 & 2.69 \\
GFRP & DPC & Whole sample & 0.35 & 0.32 \\
& AC & & 0.58 & 0.53 \\
\hline
\end{tabular}

$\mathrm{CFRP} / \mathrm{Cu}^{*}$ are supplementary results for the comparison of $\mathrm{AC}$ and DPC porosities in regions where the AC data is not affected by metal artefacts
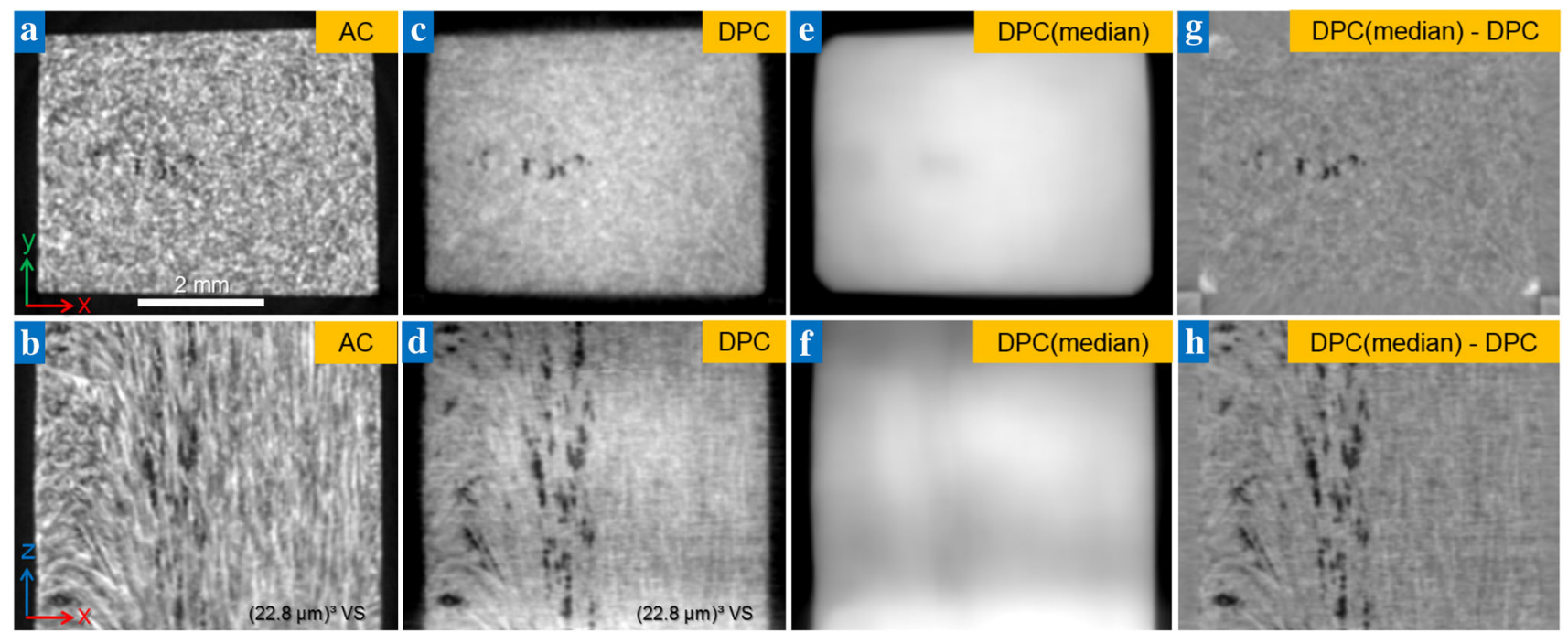

Fig. 5 Input data and post-processing of GFRP sample: $\mathbf{a}-\mathbf{h}$ axial and sagittal slice images of AC and DPC as input data with $\mathbf{e}-\mathbf{h}$ post-processing steps including $27 \times 27 \times 27$ median filtering and the

calculation of a difference image between original DPC and median filtered DPC data in order to get rid of the intensity variations, especially visible in the sagittal slice image of DPC in $\mathbf{d}$

applying a global threshold technique to certain regions of interest (entire sample area and regions close to the copper mesh-around $0.2 \mathrm{~mm}$ ). The global threshold was determined by manually selecting sample regions and defining appropriate grey values regions corresponding to areas of pores and matrix material to calculate the ISO50 threshold value. The image Fig. $4 \mathrm{~b}$ shows pores with a total defect volume of $11.1 \mathrm{~mm}^{3}$ (2.43\% porosity) and the image Fig. 4c only pores near the copper mesh with a defect volume of $0.42 \mathrm{~mm}^{3}(0.09 \%$ porosity). Reduced artefacts and improved image quality of DPC data, especially near the copper mesh, may improve the accuracy of the porosity inspection task. A porosity volume of $0.42 \mathrm{~mm}^{3}$ was quantified in a region close to the metallic components that may be missed if only AC data is evaluated, since these defects are concealed in the AC modality by strong artefacts in the form of dark streaks between the metallic wires. Porosity values are summarized in Table 2. Additionally, Table 2 provides porosity

values for the comparison of $\mathrm{AC}$ and $\mathrm{DPC}\left(\mathrm{CFRP} / \mathrm{Cu}^{*}\right)$ in regions where $\mathrm{AC}$ is not prone to metal artefacts. This artefact affected regions are excluded, since the dark streaks close to the copper mesh would be falsely interpreted as pores during segmentation and would finally lead to very high porosity values. Further, the height of the evaluated region is limited to $9 \mathrm{~mm}$, which is the scanned height of the AC scan. The resulting difference in porosity from $2.69 \%$ (DPC) to $3.09 \%$ (AC) for this sample is caused by very small pores that are beyond the detection limit of the SkyScan device, even though pores detected by DPC tend to be slightly bigger than in the high-resolution AC scan.

\subsection{Glass Fibre Reinforced Polymer (GFRP)}

Figure 5a-h shows axial (top row) and sagittal slice images (bottom row) with subsequent image processing results (Fig. 5e-h) that are needed for porosity evaluation. 


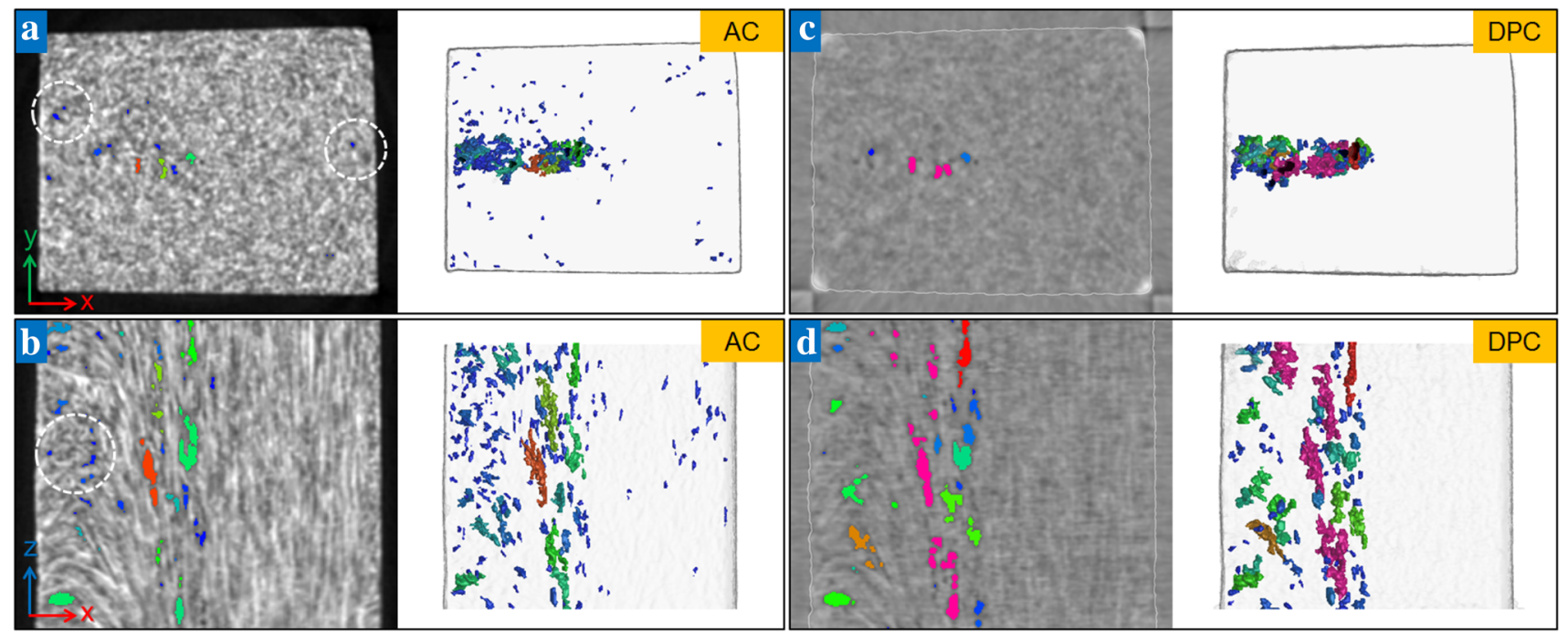

Fig. 6 Segmentation results of GFRP sample $\mathbf{a}, \mathbf{b}$ AC and $\mathbf{c}, \mathbf{d}$ difference DPC image data with corresponding 3D renderings of segmented pores along different directions, the colour coding corresponds to the volume of the pores (Color figure online)

In comparison to $\mathrm{AC}$ data, the glass fibres are less distinguishable from their matrix material in DPC data, which is due to similar refraction properties and similar propagation speed of X-rays in the case of polypropylene and glass fibres. In addition, DPC shows a good contrast between pores and matrix material that facilitates the porosity evaluation task in the case of the GFRP sample. Nevertheless, the sagittal DPC image data shows intensity variations along the $\mathrm{z}$ direction (rotational axis), due to remaining Moiré pattern that could not be completely eliminated by the data extraction process. Moiré fringes are periodic intensity modulations due to the Moiré effect that arise when two nearly identical periodic structures are superimposed. Grating imperfections due to the manufacturing process, slight misalignments between the gratings and thermal stability issues of the system (X-ray tube, gratings) may lead to this kind of intensity variations.

In order to overcome this particular problem which may have an impact on pore segmentation, a combination of simple image processing steps (high-pass filtering) was employed by first applying a $27 \times 27 \times 27$ median filter to the DPC data followed by the calculation of a difference DPC image between unfiltered and median filtered DPC data. The median filtering results in a heavily blurred image in which all pores are smeared out while the intensity modulation due to the Moire pattern is preserved. The calculation of the difference DPC image has the effect that the unwanted intensity modulations are subtracted from the original DPC data, leading to a new image that only shows the contribution of pores. Due to the subtraction process the information on the sample geometry is lost. Note that corners are smeared out strongly leading to white corners in the subtracted sample volume. This high-pass filtering allows the use of simple thresholding, but marginally reduces the contrast between pores and back- ground, since the subtracted background contains at least a very blurred representation of pores. Therefore, high-pass filtering would be not as beneficial for AC scans, since AC data is not prone to wide-ranging intensity variations related to artefacts. The XCT slice images in Fig. $6 c$, d show a white outline, indicating the outer sample geometry, which has been calculated by the surface determination tool in VGStudio MAX 3.0 and which is used as a region of interest for further data evaluations. Figure $6 \mathrm{a}, \mathrm{b}$ show the segmentation results of the $\mathrm{AC}$ as slice images and 3D renderings. Figure $6 \mathrm{c}, \mathrm{d}$ show the segmentation results of the difference DPC image data. In both cases a global threshold has been applied to the data by manually selecting sample regions and defining appropriate grey values regions corresponding to areas of pores and the subtracted background to calculate the ISO50 threshold value. In the case of AC a defect volume of about $0.35 \mathrm{~mm}^{3}$ is determined, which corresponds to a porosity value of $0.32 \%$, whereas the DPC's segmentation result suggests a larger defect volume of about $0.58 \mathrm{~mm}^{3}$, which corresponds to a porosity of $0.53 \%$. The results are visualized as 3D images in Fig. 6a-d, where the colour coding corresponds to the volume of the pores. Porosity values are summarized in Table 2 .

When taking the DPC modality again as the basis for the position of potential defects, the segmentation results of AC data show a contribution of small areas (see white dotted circles and 3D rendering in Fig. 6a, b) that might not belong to the real porosity value and should not be considered as small pores, but rather correspond to areas with less fibre content or to streaking artefacts from the higher absorbing glass fibres. The DPC modality tends to detect larger pore sizes compared to the AC modality, since the background (matrix material) is more homogeneous, which eases the detection of pores. 
Overall this leads to higher porosity values detected in DPC data.

\section{Conclusions and Outlook}

The possibilities and advantages of TLGI-based phase contrast imaging applied to fibre-reinforced polymers, expanding the application areas of Talbot-Lau imaging for material sciences were demonstrated. The main reason DPC has several advantages and better image quality for the characterization of multi-material systems is a rather linear dependence of X-ray energy/atomic number and the refraction angle and subsequent differential phase contrast. Therefore, DPC is less prone to beam hardening effects especially for multi-material systems with large differences in attenuation coefficients. This results in a better contrast between air and plastic material even in the presence of metal components. Both material systems were evaluated regarding their porosity.

The DPC modality provides less streaking artefacts from high-absorbing components as well as a good trade-off between contrast, noise and image sharpness, thus greatly improving the detection capabilities of pores within the CFRP with copper and GFRP material systems without needing further MAR correction methods. Nevertheless, DPC is lacking in sharpness and tends to blur and overestimate the actual pore size, since Talbot-Lau imaging is usually performed with larger focal spot sizes to compensate for the method's longer measurement times. This overestimation is even more emphasized by applying further image-processing as is the case with the GFRP sample, in which median filtering is required for the generation of a difference image in order to avoid the Moiré pattern. Although DPC can be seen as fundamental in helping to visually identify areas of pores, the actual porosity values have to be further evaluated quantitatively by e.g. materialographic sectioning and acid digestion of actual samples or by measurements of specially-prepared and calibrated porosity phantoms similar to B. Plank et al. [30]. Even though the three imaging modalities AC, DPC and DFC separate different material phases, properties and features, it might also be useful to combine these imaging modalities into one single image to show all material related features at once. The proposed image fusion concept of all three modalities enables the detection and direct comparison of pores with respect to the positions of the detected fibre bundles.

One major drawback of TLGI systems is the high scanning effort that is needed to extract reasonable data quality. Since statistical iterative reconstruction algorithms are already applicable for larger datasets [31], typically in the case of industrial XCT scanning, these algorithms may help to reduce the scanning effort by e.g. significantly reducing the number of projection images during data acquisition. The promising results of DPC applied to multi-material systems and future developments may increase the relevance of DPC for further material science applications and raise the acceptance and technological relevance from currently ongoing basic research to an increasing industrial use. This holds true especially for improving the inspection of lightening conduction structures. DPC modality has a high potential to supplement 2D standard methods such as optical microscopy to investigate regions close to the copper mesh for additional defects such as micro cracks [32] in 3D, since conventional AC modality is not offering sufficient detection certainty as it is shown in this contribution.

Acknowledgements Open access funding was provided by University of Applied Sciences Upper Austria. This work was supported by the KProject for "non-destructive testing and tomography plus" (ZPT+) and by the COMET program of FFG and the federal government of Upper Austria and Styria and supported by the Project "multimodal and in situ characterization of inhomogeneous materials" (MiCi) by the federal government of Upper Austria and the European Regional Development Fund (EFRE) in the framework of the EU-Program IWB2020.

Open Access This article is distributed under the terms of the Creative Commons Attribution 4.0 International License (http://creativecomm ons.org/licenses/by/4.0/), which permits unrestricted use, distribution, and reproduction in any medium, provided you give appropriate credit to the original author(s) and the source, provide a link to the Creative Commons license, and indicate if changes were made.

\section{References}

1. David, C., Nöhammer, B., Solak, H.H., Ziegler, E.: Differential xray phase contrast imaging using a shearing interferometer. Appl. Phys. Lett. 81, 3287-3289 (2002)

2. Momose, A., Kawamoto, S., Koyama, I., Hamaishi, Y., Takai, K., Suzuki, Y.: Demonstration of X-ray talbot interferometry. Jpn. J. Appl. Phys. 42, 866-868 (2003)

3. Pfeiffer, F., Weitkamp, T., Bunk, O., David, C.: Phase retrieval and differential phase-contrast imaging with low-brilliance X-ray sources. Nat. Phys. 2, 258-261 (2006)

4. Rutishauser, S., Bednarzik, M., Zanette, I., Weitkamp, T., Börner, M., Mohr, J., David, C.: Fabrication of two-dimensional hard X-ray diffraction gratings. Microelectron. Eng. 101, 12-16 (2013)

5. Fu, J., Hu, X., Velroyen, A., Bech, M., Jiang, M., Pfeiffer, F.: 3D algebraic iterative reconstruction for cone-beam X-ray differential phase-contrast computed tomography. PLoS One. 10(3), e0117502 (2015). https://doi.org/10.1371/journal.pone.0117502

6. Scholkmann, F., Revol, V., Kaufmann, R., Baronowski, H., Kottler, C.: A new method for fusion, denoising and enhancement of $\mathrm{x}$ ray images retrieved from Talbot-Lau grating interferometry. Phys. Med. Biol. 59, 1425-1440 (2014)

7. Revol, V., Plank, B., Kaufmann, R., Kastner, J., Kottler, C., Neels, A.: Laminate fibre structure characterisation of carbon fibrereinforced polymers by X-ray scatter dark field imaging with a grating interferometer. NDT\&E Int. 58, 64-71 (2013)

8. Yang, F., Prade, F., Griffa, M., Jerjen, I., Di Bella, C., Herzen, J., Sarapata, A., Pfeiffer, F., Lura, P.: Dark-field X-ray imaging of unsaturated water transport in porous materials. Appl. Phys. Lett. 105, 154105 (2014)

9. Hannesschläger, C., Revol, V., Plank, B., Salaberger, D., Kastner, J.: Fibre structure characterisation of injection moulded short fibre- 
reinforced polymers by X-ray scatter dark field tomography. Case Stud. Nondestruct. Test. Eval. 3, 34-41 (2015)

10. Gusenbauer, C., Leiss-Holzinger, E., Senck, S., Mathmann, K., Kastner, J., Hunger, S., Birkfellner, W.: Characterization of medical and biological samples with a Talbot-Lau grating interferometer $\mu \mathrm{XCT}$ in comparison to reference methods. Nondestruct. Test. Eval, Case Stud (2016). https://doi.org/10.1016/j.csndt.2016.02.0 01

11. Revol, V., Jerjen, I., Kottler, C., Schütz, P., Kaufmann, R., Lüthi, T., Sennhauser, U., Straumann, U., Urban, C.: Sub-pixel porosity revealed by x-ray scatter dark field imaging. J. Appl. Phys. 110, 044912 (2011)

12. Lauridsen, T., Willner, M., Bech, M., Pfeiffer, F., Feidenhans, R.: Detection of sub-pixel fractures in X-ray dark-field tomography. Appl. Phys. A 121(3), 1243-1250 (2015)

13. Bech, M., Bunk, O., Donath, T., Feidenhans'1, R., David, C., Pfeiffer, F.: Quantitative x-ray dark-field computed tomography. Phys. Med. Biol. 55(18), 5529-5539 (2010)

14. Prade, F., Schaff, F., Senck, S., Prade, F., Pfeiffer, F.: Nondestructive characterization of fiber orientation in short fiber reinforced polymer composites with X-ray vector radiography. NDT and E Int. 86, 65-72 (2017)

15. Malecki, A., Potdevin, G., Biernath, T., Eggl, E., Willer, K., Lasser, T., Maisenbacher, J., Gibmeier, J., Wanner, A., Pfeiffer, F.: X-ray tensor tomography. EPL 105, 38002 (2014)

16. Gresil, M., Revol, V., Kitsianos, K., Kanderakis, G., Koulalis, I., Sauer, M., Trétout, H., Madrigal, A.: EVITA Project: comparison between traditional non-destructive techniques and phase contrast $\mathrm{x}$-ray imaging applied to aerospace carbon fibre reinforced polymer. Appl. Compos. Mater. 24(2), 513-524 (2017)

17. Stampanoni, M., Wang, Z., Thüring, T., David, C., Rössl, E., van Stevendaal, U., Köhler, T., Trippel, M., Singer, G., Kubik-Huch, R.A., Hohl, M.K., Hauser, N.: Toward clinical differential phase contrast mammography: preliminary evaluations and image processing schemes. J. Instrum. (2013). https://doi.org/10.1088/17480221/8/05/C05009

18. Kastner, J., Plank, B., Salaberger, D., Sekelja, J.: Defect and Porosity Determination of Fibre Reinforced Polymers by X-ray Computed Tomography, 2nd International Symposium on NDT in Aerospace, Hamburg, Germany, 2010

19. Gagné, M., Therriault, D.: Lightning strike protection of composites. Prog. Aerosp. Sci. 64, 1-16 (2014)

20. Talbot, H.F.: Phil. Mag. 3(9), 401-407 (1836)

21. Lau, E.: Annual Physics 437, 417-423 (1948)
22. Yashiro, W., Terui, Y., Kawabata, K., Momose, A.: On the origin of visibility contrast in $\mathrm{x}$-ray Talbot interferometry. Opt. Express 18(16), 16890-16901 (2010)

23. Gusenbauer, C., Hunger, S., Senck, S., Kastner, J.: Characterization of Tooth Samples with a Talbot-Lau Grating Interferometer $\mu$ XCT Desktop Device, Proceedings of 4th International Conference on Image Formation in X-Ray Computed Tomography, Bamberg, Germany (2016)

24. Gusenbauer, C., Reiter, M., Plank, B., Senck, S., Hannesschläger, C., Renner, S., Kaufmann, R., Kastner, J.: Multi-modal Talbot-Lau grating interferometer XCT data for the characterization of carbon fibre reinforced polymers with metal components, Proceedings of 7th Conference on Industrial Computed Tomography, Leuven, Belgium (2017)

25. Pfeiffer, F., Bech, M., Bunk, O., Kraft, P., Eikenberry, E.F., Brönnimann, C., Grünzweig, C., David, C.: Hard-X-ray dark-field imaging using a grating interferometer. Nat. Mater. 7, 134-137 (2008)

26. Kalender, W.A., Hebel, R., Ebersberger, J.: Reduction of CT artifacts caused by metallic implants. Radiology 164(2), 576-577 (1987)

27. Meyer, E., Raupach, R., Lell, M., Schmidt, B., Kachelrieß, M.: Normalized metal artifact reduction (NMAR) in computed tomography. Med. Phys. 37, 5482-5493 (2010)

28. Gusenbauer, C., Reiter, M., Salaberger, D., Kastner, J.: Comparison of Metal Artefact Reduction Algorithms from Medicine Applied to Industrial XCT Applications, Proceedings of 19th World Conference on Non-Destructive Testing 2016, Munich, Germany (2016)

29. Momose, A., Takeda, T., Itai, Y., Hirano, K.: Phase-contrast X-ray computed tomography for observing biological soft tissues. Nat. Med. 2(5), 596 (1996)

30. Plank, B., Rao, G., Kastner, J.: Evaluation of CFRP-Reference Samples for Porosity made by Drilling and Comparison with Industrial Porosity Samples by Means of Quantitative X-ray Computed Tomography-Proceedings 7th International Symposium for NDT in Aerospace, pp. 10. Bremen, Deutschland (2015)

31. Fehringer, A., Mechlem, K., Epple, M., Allner, S., Hehn, L., Pfeiffer, F., Noël, P.B.: Ultra-fast cone-beam SIR on 2 k-cubed data, The 4th International Conference on Image Formation in X-Ray Computed Tomography, Bamberg, Germany (2016)

32. Kawakami, H., Feraboli, P.: Lightning strike damage resistance and tolerance of scarf-repaired mesh-protected carbon fiber composites. Composit. Part A 42, 1247-1262 (2011) 